\title{
The imagery effect and frequency discrimination
}

\author{
GEORGE D. GOEDEL and CAROL A. THOMAS \\ State University of New York, College at Geneseo, Geneseo, New York 14454
}

\begin{abstract}
A 300-item list of concrete and abstract nouns with varying frequency of occurrence was presented at a 1-sec rate to 144 subjects under imagery or nonimagery instructions. Subjects were then tested on 72 word pairs homogeneous and heterogeneous with respect to concreteness, where the more frequent member of each pair was to be chosen. Frequency discrimination was found to be a function of relative rather than absolute frequency differences between pair members. In addition, a subjective frequency bias for abstract items was found, with the best performance when the more frequent alternative was abstract and less frequent alternative concrete. The worst performance was for the reverse condition, while that for pair types homogeneous with respect to concreteness fell in-between, with better performance when both alternatives were concrete. The results suggest that the role of imagery may be to produce more discriminable subjective relative frequency differences between alternatives and that the imagery effect generally found in verbal discrimination learning may be reconcilable with frequency theory as it currently stands.
\end{abstract}

One of the more reliable findings concerning verbal discrimination learning (VDL) over the last few years has been the so-called "imagery effect" when concrete and abstract word materials are employed. Differential performance on word pairs homogeneous with respect to concreteness (i.e., image-arousal capacity) is typically found with faster learning when both members of a pair are concrete rather than abstract in mixed as well as unmixed list designs (Paivio \& Rowe, 1970, 1971; Rowe \& Paivio, 1971, 1972; Ullich \& Balogh, 1972).

An attempt was initially made (Paivio \& Rowe, 1970) to integrate this phenomenon within the general theoretical framework of frequency theory (Ekstrand, Wallace, \& Underwood, 1966; Wallace, 1972) which has thus far served well to explain VDL. This attempt took the form of assuming implicit imaginal response evocation to correct high-imagery items more than to incorrect high-imagery items and differential frequency accrual analogous to that postulated by frequency theory for implicit associative responses (IARs). Subsequent failures to confirm several predictions based upon this assumption led to the abandonment of frequency theory interpretations in favor of image "tagging" or differential learning strategy interpretations (Rowe \& Paivio, 1971 ) and to the conclusion that imagery and frequency are independent processes in VDL (Rowe, 1972a, b).

It now seems that the abandonment of frequency theory may have been somewhat premature. Wallace, Murphy, and Sawyer (1973), in a recent monograph, pointed out that the critical requirement for a frequency theory interpretation of the imagery effect is a demonstration that correct and incorrect alternatives of concrete word pairs are more discriminable in terms of

Requests for reprints should be sent to George D. Goedel, Department of Psychology, State University of New York, College at Geneseo, Geneseo, New York 14454. subjective frequency than are alternatives of abstract word pairs. Using a comparative frequency judgment task in their final experiment, they found frequency discriminations for concrete word pairs consistently easier than those for abstract word pairs across several presentation frequency contrasts.

Rowe (1975) initially acknowledged the difficulty such results presented to the idea that the imagery effect is independent of frequency in VDL. However, he minimized their relevance, arguing that it is difficult to equate the processes underlying frequency judgment and VDL tasks. This argument is based on the fact that subjects are required to make subjective frequency decisions based on responses to items differing in objective presentation frequency in a comparative judgment task, whereas subjective frequency decisions in VDL are based on responses to items having the same objective presentation frequency. It would seem, however, that decisions based upon subjective frequency are involved in both tasks. Furthermore, if the accrual of subjective frequency units for concrete items differs from that for abstract items in such a manner that frequency discriminations are easier for concrete pairs than abstract pairs (in a relative rather than absolute sense) in a paired comparison test, it would be parsimonious to infer similar differences in subjective frequency accrual for these items in VDL.

One of the purposes of the present investigation was an attempt to replicate and extend the Wallace et al. (1973) findings using a factorial manipulation of presentation frequency and a comparative frequency judgment task of word pairs homogeneous and heterogeneous with respect to item concreteness. A second purpose was to explore a possible interpretation as to why frequency discriminability between alternatives of concrete word pairs is better than that for abstract word pairs consistent with frequency theory as it 
presently stands. This interpretation rests on the assumption that frequency discrimination is a function of relative rather than absolute frequency differences as predicted by the Weber law postulate of frequency theory and that higher subjective frequencies are given to abstract relative to concrete word items with identical objective presentation frequencies.

Available evidence with experimental frequency would support the notion that frequency discrimination is based on relative rather than absolute frequency differences (Hintzmann, 1969; Radtke, Jacoby, \& Goedel, 1971; Underwood \& Freund, 1970), consistent with the Weber law postulate. Also, recent evidence has indicated greater perceived frequency for abstract than concrete words when either objective background frequency is equal based upon available word counts (Galbraith \& Underwood, 1973) or when objective experimental frequency is equal for low-presentation frequencies and delayed judgments (Begg, 1974). Therefore, if subjective frequency of occurrence for both members of an abstract word pair is greater than that for members of a concrete word pair of equal objective presentation frequency, the Weber law postulate of frequency theory would predict poorer performance on the former due to the less discriminable subjective relative frequency difference between pair members.

Support for such an interpretation in the present experiment could come from two sources. First, the manipulation of objective presentation frequency should interact with pair type tested: An increase in frequency for the correct or incorrect member of a pair would have greater effect on frequency discrimination of homogeneous concrete word pairs if subjective frequency of abstract items is indeed higher than that of concrete items. Second, frequency discrimination of word pairs heterogeneous with respect to concreteness should be enhanced when the more frequent (or correct) item of the pair is abstract, and depressed when the more frequent (or correct) item is concrete, relative to word pairs homogeneous with respect to concreteness due to a subjective frequency bias in favor of abstract items.

\section{METHOD}

\begin{abstract}
Design
The design of the experiment was a 2 by 3 by 3 by 4 mixed factorial with two levels of study instructions (encouraged to use imaginal encoding vs. no reference to imaginal encoding) as the between-subjects factor. The three within-subjects factors included frequency of occurrence during study of the more frequent or correct member of a test pair $(F C=2,3$, or 4$)$, frequency of occurrence during study of the less frequent or incorrect member of a test pair $(F I=0,1$, or 2$)$, and the type of pair tested $(\mathrm{PT}=$ both members concrete, $\mathrm{CC}$; both members abstract, $A A$; the more frequent member concrete and less frequent member abstract, $\mathrm{CA}$; and the more frequent member abstract and less frequent member concrete, $\mathrm{AC}$ ).
\end{abstract}

\section{Materials}

Verbal materials consisted of 72 concrete (C) and 72 abstract
(A) words drawn from the Spreen and Schulz (1966) norms, with mean ratings of 6.61 and 2.48 , respectively, on a 7-point scale. All words had T-L frequency values of A-AA with interitem associations minimized. Care was also taken to match $\mathrm{m}$ values as closely as possible between the two classes of words. Half of the $\mathrm{C}$ and $\mathrm{A}$ words were randomly selected as the correct or more frequently occurring alternative of a test trial pair (two, three, or four occurrences during study), with the remaining words as incorrect (zero, one, or two occurrences during study). Thus, the frequency manipulation was a 3 by 3 factorial with eight correct ( $4 A$ and $4 C$ ) and eight incorrect (4A and $4 C$ ) items in each of the nine cells. Within a cell, four types of pairs were tested (CC, AA, CA, and AC), with two pairs per PT condition. Twelve additional words, neutral with respect to concreteness, served as primacy (five) and recency (seven) buffers, yielding a study list of 300 consecutive items.

Two random orders of the study list were constructed. Across six replications of each, every $\dot{C}$ and $A$ word was rotated through the six correct and incorrect frequency levels. Repetitions of a word within a list were separated by not less than 7 nor more than 11 other items (average lag of 9), with no two words in any list ever occurring more than once in adjacent succession. The initial and final occurrence of half of the $\mathrm{C}$ and A words occurred in each half of the study list.

There were three random orders of the test list where correct or more frequently appearing study items were paired with different incorrect or less frequently occurring study items of the appropriate concreteness and frequency level. One pair under each of the 36 FC by FI by PT conditions was tested before the second pair of any condition was tested. The correct or more frequent item of a pair was on the left for half the pairs and on the right for the other half.

\section{Procedure}

A total of 300 consecutive study trial items were presented individually on a memory drum at a $1-\mathrm{sec}$ rate. Each subject was required to pronounce aloud each word as it appeared in the window of the drum. Subjects were informed that there were 300 study items, frequency of occurrence of items varied, and their task following study would be to circle the more frequently occurring member of each of 72 word pairs. Half of the subjects were told, in addition, that some words were concrete and were encouraged to try to form mental images during study of the objects to which these words referred.

Following the presentation of the study list, each subject received a two-page booklet containing 72 test trial pairs, with two columns of 18 pairs per page. Subjects circled one member of each pair at their own pace-the one they felt had occurred more frequently on the study trial. All subjects completed study and test in less than $15 \mathrm{~min}$.

\section{Subjects}

The subjects were 144 students at the State University of New York at Geneseo enrolled in introductory psychology classes. The subjects received course credit for their voluntary participation. None of the subjects had prior experience in verbal learning studies. They were assigned randomly to instruction and list conditions as they appeared in the laboratory, with the restriction that the $n+1$ subject was not assigned until the $n$th subject had been tested in every condition.

\section{RESULTS}

The basic data subjected to a 2 by 3 by 3 by 4 mixed analysis of variance were the number of incorrect identifications of the more frequent alternative (errors), which could range from zero to two for each condition for each subject. The analysis revealed no significant 
Table 1

Mean Total Errors on Test Pairs as a Function of Frequency Level of Correct and Incorrect Alternatives During Study

\begin{tabular}{ccccc}
\hline & \multicolumn{4}{c}{ Frequency Correct } \\
\cline { 2 - 5 } Frequency & 2 & 3 & 4 & $\overline{\mathrm{X}}$ \\
\hline 0 & .39 & .39 & .34 & .37 \\
1 & .73 & .59 & .51 & .61 \\
2 & 1.01 & .79 & .68 & .83 \\
$\overline{\mathrm{X}}$ & .71 & .59 & .51 & \\
\hline
\end{tabular}

effect of instructions nor any interactions involving this variable.

The main effect of $\mathrm{FC}[\mathrm{F}(2,284)=51.35]$, FI $[F(2,284)=238.05]$, and their interaction ( $F C$ by $F I)$ $[F(4,568)=8.97]$ were all significant at $p<.01$ $(\mathrm{MSe}=.37, .37$, and .36 , respectively). Table 1 presents the mean errors collapsed over pair type and instructions.

It can be seen that the effect of FC increases as FI increases, while the effect of FI decreases as FC increases. Upon inspection of the cells in the left-to-right diagonals, where absolute frequency differences between pair members remain the same while relative frequency differences decrease (e.g., main diagonal: $2: 0,3 ; 1$, and $4: 2$ ), a decrease in performance can be noted. All possible pairwise comparisons of the diagonal means proved significant at the .01 level using Dunn's multiple comparison test (Kirk, 1969). In those cells where relative frequency remains unchanged $(2: 1$ and $4: 2)$, although absolute frequency differences change, comparable levels of performance were obtained (.73 and .68 mean errors, respectively). Furthermore, it should perhaps be noted that performance was near chance for Cell $2: 2$, as would be expected if subjects were responding on the basis of frequency, since $F C$ equaled FI in this cell. In general, these data support a relative rather than absolute hypothesis as the basis for frequency discrimination consistent with the Weber law postulate of frequency theory.

A significant effect was also found for PT

Table 2

Mean Total Errors for the Four Pair Types Tested as a Function of Frequency During Study of Correct Alternative and Incorrect Alternative

\begin{tabular}{ccccc}
\hline \multirow{2}{*}{$\begin{array}{c}\text { Frequency } \\
\text { Alternative }\end{array}$} & $\mathrm{CC}$ & $\mathrm{CA}$ & $\mathrm{AC}$ & $\mathrm{AA}$ \\
\hline \multicolumn{5}{c}{ Correct } \\
2 & .72 & .96 & .47 & .70 \\
3 & .50 & .80 & .40 & .65 \\
4 & .41 & .69 & .37 & .54 \\
& \multicolumn{5}{c}{ Incorrect } \\
0 & .28 & .56 & .18 & .48 \\
1 & .54 & .88 & .43 & .59 \\
2 & .81 & 1.02 & .64 & .83 \\
$\overline{\mathrm{X}}$ & .54 & .82 & .41 & .63 \\
\hline
\end{tabular}

$[\mathrm{F}(3,426)=73.60, \mathrm{p}<.01, \mathrm{MSe}=.50]$ as well as for the interactions of this variable with $F C[F(6,852)=3.26$, $\mathrm{MSe}=.34]$ and FI $[\mathrm{F}(6,852)=2.97, \mathrm{MSe}=.38]$ (both ps $<.01$ ). These results are given in Table 2 .

Clearly, the best performance was obtained when the correct member of a pair was abstract and the incorrect member concrete (AC), followed by $\mathrm{CC}, \mathrm{AA}$, and $\mathrm{CA}$. All possible Tukey comparisons of these pair types proved significant at the .01 level. These results indicate a clear bias, in terms of subjective frequency, for abstract items. This bias can also be found by noting performance in the 2:2 cell (not given in table) for CA and $\mathrm{AC}$. Although chance performance (mean errors $=$ 1.00) would be expected in this cell if subjects were responding on the basis of equal presentation frequency for $\mathrm{C}$ and $\mathrm{A}$ items, mean errors were 1.19 for $\mathrm{CA}$ and .75 for $\mathrm{AC}$. This data would further suggest subjective frequency bias for abstract items.

The PT by FC interaction (see Table 2) shows the effect of FC to be greater in those pairs where concrete members were correct ( $\mathrm{CC}$ and $\mathrm{CA}$ ) relative to pairs where abstract members were correct (AA and $\mathbf{A C}$ ). If one assumes higher subjective frequency for abstract items, such results would be expected, since increasing the objective frequency of a correct member when its subjective frequency is high should have less effect on relative frequency discrimination than when it is low. The effect of FI as a function of PT (see Table 2), although somewhat less clear, reveals a similar pattern, being strongest for $\mathrm{CC}$ pairs and weakest for AA pairs. Again, the manipulation of incorrect objective frequency should have less effect when subjective frequency is high rather than low.

Although the critical three-way interaction of PT by FC by FI failed to reach significance, it is of some interest to examine the FC by FI interactions for the CC and AA pair types as given in Table 3. It appears from this table that the objective relative frequency manipulations (see main diagonals: 2:0,3:1, and 4:2) are somewhat more effective for CC than AA. Upon closer inspection of this data, two potential sources of difficulty might appear evident. The first is the mean error score (1.10) obtained in the $2: 2$ cell for the CC condition, where performance would be expected to be closer to chance and the better than expected performance in the $3: 2$ cell (relative to the $2: 1$ cell) of the same condition. Inspection of the raw data for these conditions across the various study and test list replications provided no clear explanation as to why such unexpected results obtained in these cells.

A second difficulty concerns the comparison of $\mathrm{CC}$ and AA conditions where objective frequency of the incorrect member was zero (never presented during study). If subjective frequency of abstract items is indeed higher than concrete, one might predict better performance on AA pairs where the objective frequency relationship was $2: 0,3: 0$, or $4: 0$ compared to perform- 
Table 3

Mean Total Errors as a Function of Frequency Level of Correct and Incorrect Alternatives for Test Pairs Homogeneous with Respect to Concreteness

\begin{tabular}{|c|c|c|c|c|c|c|c|c|}
\hline \multirow{2}{*}{$\begin{array}{l}\text { Frequency } \\
\text { Incorrect }\end{array}$} & \multicolumn{4}{|c|}{$\begin{array}{c}\text { Pair Type CC } \\
\text { Frequency Correct }\end{array}$} & \multicolumn{4}{|c|}{$\begin{array}{c}\text { Pair Type AA } \\
\text { Frequency Correct }\end{array}$} \\
\hline & 2 & 3 & 4 & $\overline{\mathbf{X}}$ & 2 & 3 & 4 & $\overline{\mathbf{X}}$ \\
\hline$\overline{\mathbf{X}}$ & .72 & .50 & .41 & & .70 & .65 & .54 & \\
\hline
\end{tabular}

ance in those cells for $\mathrm{CC}$ pairs. Since the opposite was found to be the case, we are led to conclude that the subjective frequency for incorrect members in these cells was above zero, with somewhat higher subjective frequency for incorrect members of AA pairs than CC pairs (possibly due to higher subjective preexperimental frequency or differential frequency generated during study).

Since a major focus of the study was the interactions of FC and FI with the homogeneous pair types, a second analysis of variance ( 2 by 3 by 3 by 2 ) was performed only on the data for the $\mathrm{CC}$ and AA pair types. This analysis yielded exactly the same effects as the larger analysis and, thus, will not be reported.

\section{DISCUSSION}

The instruction variable, which was of some interest in the present study, failed to yield any significant effects. This manipulation was included in the design since it was felt that explicit instructions to imaginally encode where possible would enhance any differences in frequency discrimination for the various pair types tested if imagery directly affected subjective frequency accrual. The failure to obtain this effect might be attributed to the relatively rapid rate of presentation (one item/sec) which was employed. However, Paivio and Begg (1971) have suggested imaginal encoding is possible with briefer exposures. A plausible alternative for the failure of this manipulation would be if imagery was also reliably used by subjects in the nonimagery instructed group to encode concrete items in the absence of explicit instructions to do so. This interpretation is made somewhat more attractive by the subjective reports of several subjects in the nonimagery instructed group, after completion of the experiment, indicating an awareness of some words being more concrete than others during study.

The present results clearly support the hypothesis that frequency discrimination is a function of relative rather than absolute frequency differences between pair members, as predicted by the Weber law postulate of frequency theory. The fact that performance is a function of the FC to FI ratio rather than of absolute difference between objective frequency of pair members is also consistent with previous research on this question (Hintzmann, 1969; Radtke, Jacoby, \& Goedel, 1971; Underwood \& Freund, 1970).

It is also evident from the data that a definite bias, in terms of subjective frequency, exists for the abstract items in heterogeneous word pairs (AC and CA). The possibility that a response bias for abstract items may be operating for these pairs, rather than a subjective frequency bias, was considered. Such a bias could explain the FI and FC interactions with these pair types but would not predict FI and FC interactions with homogeneous pairs. Since the second analysis, which was performed only on the data for $\mathrm{CC}$ and AA pair types, showed such interactions to be present, an interpretation favoring subjective frequency bias rather than a response bias for abstract items in heterogeneous pairs seemed appropriate.

The present results also indicate that frequency discriminations are easier for homogeneous word pairs when both members of the pair are concrete than when they are abstract. These results are entirely consistent with those obtained by Wallace et al. (1973), who also employed paired comparison tests for frequency discrimination, and lend support to their argument that imagery affects situational subjective frequency in a manner that makes frequency differences between members of highimagery pairs easier to detect.

A possible explanation of this effect could be made if one returns to the fact that frequency discrimination seems to be based upon subjective relative frequency. If one is willing to assume higher subjective frequency for both members of AA pairs relative to that for $\mathrm{CC}$ pairs with the same objective presentation frequency (as suggested by the bias for $A$ items in heterogeneous pairs), more difficult subjective relative frequency differences would result between alternatives of AA pairs, and poorer performance would be expected relative to CC pairs. Such an interpretation would be consistent with the results of the present experiment (the PT by FC and PT by FI interactions) as well as with frequency theory.

An obvious source of subjective frequency bias for abstract items is that of preexperimental subjective frequency (Galbraith \& Underwood, 1973). Since such frequency was not controlled in the present study, a 
subsequent investigation of 100 new subjects utilizing the Galbraith and Underwood (1973) procedures for the words employed (plus 56 additional filler items) was undertaken. Essentially, this involved the presentation of 200 items in booklet form (two columns of 25 items/page) to each subject. To the left of each word was a short line for the subjects to record a number (1-9) to indicate how frequently they thought each word appeared in printed form. Four anchor words (the same used by Galbraith and Underwood) were given. The data essentially replicated the Galbraith and Underwood findings, yielding means of 4.55 and 5.51 for the concrete and abstract items, respectively. Although this finding is initially attractive to explain the present results, it must be tempered somewhat by the necessity to assume that subjective background frequency combines with situational in frequency discriminations. Unfortunately, the evidence suggesting such assimilation of background and experimental frequency has been rather weak and limited as given in extensive reviews by Eckert and Kanak $(1974$, p. 585) and Wallace (1972, p. 292). Therefore, although the Weber law postulate of frequency theory has received considerable support when item frequency is experimentally manipulated, there is very little support for its application with respect to background frequency.

A possible alternative source of subjective frequency bias for abstract items is that of differential frequency accrual to abstract and concrete items during study through differential rehearsal and/or IARs. It is noteworthy that Galbraith and Underwood (1973), in their discussion of differential subjective background frequency, considered greater "contextual variety" for abstract items as a possible critical factor. The concept of greater contextual variety, it would seem, should also suggest the likelihood of more and varied IARs for abstract items as well, thereby contributing to higher subjective situational frequency.

It should be remembered that Paivio and Rowe (1970) initially attempted to explain the superiority of high-imagery pairs in the VDL paradigm in terms of frequency theory. They suggested, at that time, that images evoked by concrete items might be analogous to IARs, thereby adding subjective frequency units to concrete items. The data of the present experiment suggest instead that perhaps the role of imagery may be the reverse, with the generation of unique images keeping the subjective frequency of concrete items low compared to that of abstract items. If imagery is an efficient and effective coding mechanism, its availability as an alternative means of encoding concrete materials might reduce implicit verbal rehearsal and/or associative responding, both of which are assumed by frequency theory to increase subjective frequency. Levin, Ghatala, and Wilder (1974) offered a similar line of reasoning to explain the superiority of picture discrimination over that of words. They have proposed that subjective frequency units associated with pictures may be more stable than that for words, yielding easier frequency discriminations for picture pairs.

Lastly, it is of some interest that Rowe and Paivio (1971) considered the possibility that the imagery effect in VDL may be due to a greater degree of associative interference among low-imagery items through the operation of IARs. They dismissed this explanation as inadequate, however, since IARs would be expected to affect the subjective frequency of correct and incorrect members of low-imagery pairs equally.

The results of the present study show frequency discrimination to be a function of relative frequency differences between pair members. If it can be assumed that discriminations on the basis of subjective relative frequency also occur during VDL, the addition of subjective frequency units to both members of a word pair, while not affecting absolute frequency difference, would affect relative frequency in a manner predicting poorer performance. Should such be the case, the hypothesis of greater associative interference among low-imagery items may be a viable one in explaining the imagery effect.

\section{REFERENCES}

BEGG. I. Estimation of word frequency in continuous and discrete tasks. Journal of Experimental Psychology, 1974, 102, 1046-1052.

ECKERT, E., \& KANAK, N. J. Verbal discrimination learning: A review of the acquisition, transfer and retention literature through 1972. Psychological Bulletin, 1974, 81, 582-607.

Ekstrand, B. R.. Wallace, W. P., \& Underwood, B. J. A frequency theory of verbal discrimination learning. Psychological Review, 1966, 73, 566-578.

Galbraith, R. C., \& Underwood, B. J. Perceived frequency of concrete and abstract words. Memory \& Cognition, 1973 . 1, 56-60.

Hintzman, D. L. Apparent frequency as a function of frequency and the spacing of repetitions. Joumal of Experimental Psychology, 1969, 80, 139-145.

KIRK, R. E. Experimental design: Procedures for the behavioral sciences. Belmont, Calif: Brooks/Cole, 1969.

Levin, J. R., Ghatala, E. S., \& Wilder, L. Picture-word differences in discrimination learning: I. Apparent frequency manipulations. Journal of Experimental Psychology, 1974, 102, 691-695.

Paivio, A., \& BEgG, I. Imagery and associative overlap in short-term memory. Journal of Experimental Psychology, $1971,89,40-45$.

Paivio, A., \& Rowe, E. J. Noun imagery, frequency, and meaningfulness in verbal discrimination. Journal of Experimental Psychology, 1970, 85, 264-269.

PaIvio, A., \& Rowe, E. J. Intrapair imagery effects in verbal discrimination and incidental associative learning. Canadian Journal of Psychology, 1971, 25, 302-312.

Radtke, R. C., JAcoby, L. L., \& Goedel, G. D. Frequency discrimination as a function of frequency of repetition and trials. Journal of Experimental Psychology, 1971, 89, 78-84.

Rowe, E. J. Imagery and frequency processes in verbal discrimination learning. Journal of Experimental Psychology, 1972, 95, 140-146. (a)

Rowe, E. J. Imagery and repetition instructions in verbal discrimination learning as a function of lag. Psychonomic Science, 1972, 27, 363-364. (b) 
RowE, E. J. The effect of imagery on rehearsal strategies in verbal-discrimination learning. American Journal of Psychology, 1975, 88, 431-442.

Rowe, E. J., \& PaIvio, A. Word frequency and imagery effects in verbal discrimination learning. Joumal of Experimental Psychology, 1971, 88, 319-326.

Rowe, E. J., \& Parvio, A. Effects of noun imagery, pronunciation, method of presentation, and intrapair order of items in verbal discrimination. Journal of Experimental Psychology, 1972, 93, 427-429.

SPREEN, O., \& Scrulz, R. W. Parameters of abstraction, meaningfulness, and pronunciability for 329 nouns. Journal of Verbal Learning and Verbal Behavior, 1966, 5, 459-468.

UlLRICH, J. R., \& BALOGH, B. A. Imagery and meaningfulness of right and wrong items in verbal discrimination learning. Psychonomic Science, 1972, 29, 68-70.
UNDERWOOD, B. J., \& FREUND, J. S. Relative frequency judgments and verbal discrimination learning. Journal of Experimental Psychology, 1970, 83, 279-285.

WALLACE, W. P. Verbal discrimination. In C. P. Duncan, L. Sechrest, \& A. W. Melton (Eds.) Human memory: Festschrift for Benton J. Underwood. New York: AppletonCentury-Crofts, 1972.

Wallace, W. P., Murphy, M. D., \& SaWyer, T. J. Imagery and frequency in verbal discrimination learning. Journal of Experimental Psychology, 1973, 101, 201-219. (Monograph)

(Received for publication February 10, 1976; revision accepted May 6, 1976.) 